\title{
Thermal and active fluctuations of a compressible bilayer vesicle
}

\author{
T. V. Sachin Krishnan, ${ }^{1,2}$ Kento Yasuda, ${ }^{2}$ Ryuichi Okamoto,${ }^{3}$ and Shigeyuki Komura ${ }^{2, \text { * }}$ \\ ${ }^{1}$ Department of Physics, Indian Institute of Technology Madras, Chennai, 600036, India \\ ${ }^{2}$ Department of Chemistry, Graduate School of Science and Engineering, \\ Tokyo Metropolitan University, Tokyo 192-0397, Japan \\ ${ }^{3}$ Research Institute for Interdisciplinary Science, Okayama University, Okayama 700-8530, Japan
}

(Dated: March 13, 2018)

\begin{abstract}
We discuss thermal and active fluctuations of a compressible bilayer vesicle by using the results of hydrodynamic theory for vesicles. Coupled Langevin equations for the membrane deformation and the density fields are employed to calculate the power spectral density matrix of membrane fluctuations. Thermal contribution is obtained by means of the fluctuation dissipation theorem, whereas active contribution is calculated from exponentially decaying time correlation functions of active random forces. We obtain the total power spectral density as a sum of thermal and active contributions. An apparent response function is further calculated in order to compare with the recent microrheology experiment on red blood cells. An enhanced response is predicted in the low-frequency regime for non-thermal active fluctuations.
\end{abstract}

\section{INTRODUCTION}

Vesicles idealized as closed two-dimensional (2D) elastic sheets serve as a simple model for complex biological cells such as the red blood cells (RBCs). Studies of vesicle fluctuations have offered significant insights into the physical properties of RBCs. In thermal equilibrium, local deformations of lipid membranes obey well-known statistics prescribed by several physical parameters [1]. Out-of-plane membrane fluctuations were measured by flicker analysis [2, 3] to obtain both static and dynamic properties such as the membrane bending modulus [4] or the wavelength-dependent relaxation rates [5].

Since the deformation energy of a lipid membrane is comparable to thermal energy, earlier theoretical works assumed that vesicle fluctuations are driven by thermal forces [6]. However, recent experiments have revealed the existence of active non-thermal fluctuations in addition to thermal fluctuations [7, 8]. Using both active and passive microrheology techniques, Turlier et al. measured the power spectral density (PSD) as well as the response function of a single RBC [9]. Moreover, they demonstrated a violation of the fluctuation dissipation theorem (FDT) due to non-thermal active forces. This breakdown of FDT seems to be caused by metabolic processes taking place inside the RBC such as the pumping action of ion channels or the growth of cytoskeletal networks [9].

Recent experiments have further clarified the functional significance of ATP-driven non-thermal fluctuations in cells [10]. It was shown that the activity of motor protein $\mathrm{F}_{1} \mathrm{~F}_{0}$-ATPase and actomyosin cytoskeleton affect the local organization as well as the elastic properties of the lipid membrane 11]. Moreover, membrane fluctuations act as a generic regulatory mechanism in cell adhesion by controlling the interaction between cadherin proteins [12].

\footnotetext{
* komura@tmu.ac.jp
}

So far, several theoretical models have been suggested to describe the statistics of membrane fluctuations in the presence of active forces. Prost and Bruinsma proposed a model for membrane ion pumps and showed that active fluctuations dominate at large wavelengths [13]. Later, Ramaswamy et al. considered a model that accounts for the coupling between the ion pump density and the membrane curvature, which can destabilize the membrane shape [14]. For membranes containing ion pumps, their time-dependent fluctuation or the mean square displacement (MSD) was later obtained by Lacoste and some of the present authors [15, 16]. Apart from ion pumps in membranes, cytoskeletal proteins also act as sources of active forces which affect the membrane fluctuation spectrum in a significant manner 17 20.

One important aspect that has been often neglected in the dynamics of a bilayer membrane is the role of intermonolayer friction and hence the membrane lateral compressibility. As a consequence of the bilayer structure, lipid monolayers are inevitably compressible in order to allow out-of-plane membrane deformations. It is known that the compression mode due to inter-monolayer friction drastically affects the bilayer dynamics $21-24]$. Recently, the present authors have investigated the relaxation dynamics of a compressible bilayer vesicle with an asymmetry in the viscosity of the inner and outer fluid medium 25]. Using the framework of the Onsager's variational principle [26], we showed that different relaxation modes are coupled to each other due to the bilayer structure of a spherical vesicle. We have also discussed the dynamics of a bilayer membrane coupled to a $2 \mathrm{D}$ cytoskeleton, for which the slowest relaxation is governed by the intermonolayer friction 27]. It was predicted that forces applied at the scale of cytoskeleton for a sufficiently long time can cooperatively excite large-scale modes.

In this paper, we discuss both thermal and active fluctuations of a compressible bilayer vesicle. Using the results of hydrodynamic theory that explicitly takes into account the intermonolayer friction [25], we consider the standard Langevin equation for the membrane deforma- 
tion and the lipid density fields neglecting hydrodynamic memory whose effects will be separately discussed in the final section. We particularly calculate the PSD matrix and discuss its frequency dependence. Thermal contribution to PSD is estimated by using the FDT, whereas active contribution is obtained by assuming that the time correlation of active random forces decays exponentially with a characteristic time scale. The total PSD for a bilayer vesicle is naturally given by the sum of thermal and active contributions. We also calculate an apparent response function that can be compared with a recent microrheology experiment on RBCs [9]. We shall argue that the apparent response is enhanced in the low-frequency regime in accordance with the experimental result.

In Sec. II we review the relaxation dynamics of a compressible bilayer vesicle based on our previous work [25]. In Sec. III we start with a coupled Langevin equation of a bilayer vesicle and discuss the properties of thermal fluctuations by using the FDT. The effects of active fluctuations are further discussed in Sec. IV by assuming exponentially decaying time correlations of active random forces. The summary and several discussions are finally given in Sec. V

\section{VESICLE RELAXATION DYNAMICS}

A vesicle is composed of two opposing layers of lipid molecules whose tails meet at the bilayer midsurface. For a nearly spherical vesicle, the membrane surface can be described by an angle-dependent radius field $r(\theta, \varphi)$. As shown in Fig. 1, we define a dimensionless deviation in radius $u$ by using the radius $r_{0}$ of a reference sphere as [4, $5]$

$$
u(\theta, \varphi)=\frac{r(\theta, \varphi)}{r_{0}}-1 .
$$

For bilayer membranes with finite thickness, bending deformation always accompanies stretching of one monolayer and compression of the other. Hence the membrane monolayers should be weakly compressible, and the local lipid density in each monolayer is allowed to vary. Let us denote the number of lipids in the outer and the inner monolayers as $N^{+}$and $N^{-}$, respectively. Since a spherical vesicle is stable only if the outer monolayer has more lipids than the inner one, we impose a condition $N^{+}>N^{-}$. We denote the reference lipid density by $\rho_{0}=\left(N^{+}+N^{-}\right) /\left(2 A_{0}\right)$ where $A_{0}=4 \pi r_{0}^{2}$. Let $\rho^{+}(\theta, \varphi)$ and $\rho^{-}(\theta, \varphi)$ be the variables representing the local lipid densities in the two monolayers. We then define dimensionless local density deviations in each monolayer as

$$
\phi^{ \pm}(\theta, \varphi)=\frac{\rho^{ \pm}(\theta, \varphi)}{\rho_{0}}-1 .
$$

Further calculations can be simplified by using the difference and the sum of the densities given by

$$
\phi^{\Delta}(\theta, \varphi)=\frac{\phi^{+}-\phi^{-}}{2}, \quad \phi^{\Sigma}(\theta, \varphi)=\frac{\phi^{+}+\phi^{-}}{2},
$$

respectively.

In order to describe the configuration of a bilayer vesicle, we make use of the three independent variables $u$, $\phi^{\Delta}$, and $\phi^{\Sigma}$. The free energy of a compressible bilayer vesicle is given by [22, 25]

$$
\begin{aligned}
F & =\int d A\left[\sigma+\frac{\kappa}{2}(2 H)^{2}+2 \lambda H \phi^{\Delta}\right. \\
& \left.+k\left[\left(\phi^{\Delta}\right)^{2}+\left(\phi^{\Sigma}\right)^{2}\right]\right]+\int d V \Delta P,
\end{aligned}
$$

where $H$ is the local mean curvature that can be expressed in terms of $u, \sigma$ is the membrane surface tension, $\kappa$ is the bending rigidity, $\lambda$ is the strength of coupling between local density and curvature, $k$ is the monolayer compressibility, and $\Delta P$ is the pressure difference between the inside and the outside of the vesicle. The first and the second integrals are performed over the area and the volume of the vesicle, respectively.

In general, the above three variables are all timedependent. Since they are all functions of $\theta$ and $\varphi$, we expand them in terms of surface spherical harmon-
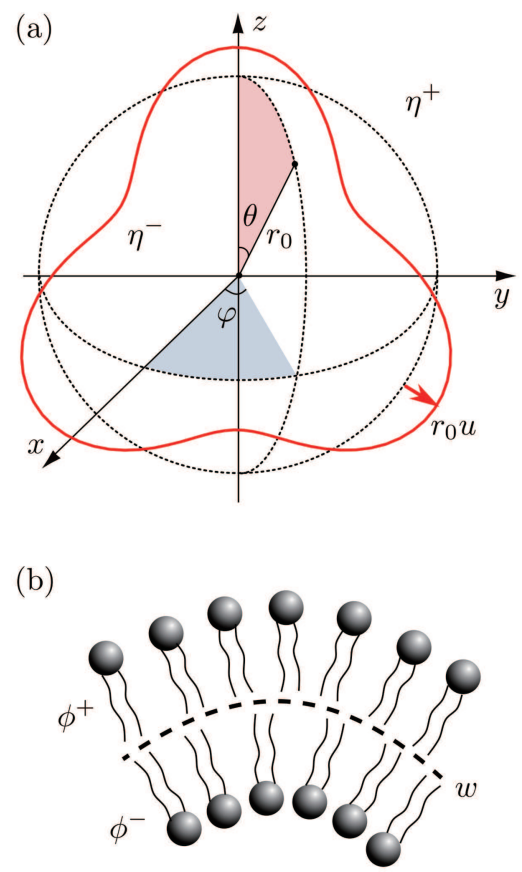

FIG. 1. (a) Schematic picture of a vesicle showing the reference sphere (dashed line) of radius $r_{0}$ with fluids of viscosity $\eta^{-}$inside and $\eta^{+}$outside. Solid red line represents a deformed vesicle configuration whose shape is parametrized with $u(\theta, \varphi)$. (b) Cross-section of a curved region in the bilayer showing the lipid molecules in each monolayer. Local densities in the monolayers are indicated as $\phi^{+}$and $\phi^{-}$which are defined on the bilayer midsurface represented by the dashed line. The inter-monolayer friction $w$ also acts at the bilayer midsurface. 
$\operatorname{ics} Y_{n m}(\theta, \varphi)$ as 22,25$]$

$$
u(\theta, \varphi, t)=\sum_{n=0}^{\infty} \sum_{m=-n}^{n} u_{n m}(t) Y_{n m}(\theta, \varphi),
$$

and

$$
\begin{aligned}
\delta \phi^{\Delta}(\theta, \varphi, t) & =\phi^{\Delta}-\phi_{0}^{\Delta} \\
& =\sum_{n=2}^{\infty} \sum_{m=-n}^{n} \phi_{n m}^{\Delta}(t) Y_{n m}(\theta, \varphi),
\end{aligned}
$$

where $\phi_{0}^{\Delta}=\left(N^{+}-N^{-}\right) /\left(N^{+}+N^{-}\right)$. It can be shown that the mode associated with $\phi^{\Sigma}$ is decoupled from the other two modes and will not be considered hereafter.
The dynamics of a vesicle is affected by the viscosities of the outside and the inside bulk fluids, $\eta^{ \pm}$[28 30$]$, and the friction constant between the two monolayers, $w$ 2125]. We have shown that the relaxation equations for $u$ and $\phi^{\Delta}$ are given by the set of coupled equations [25]

$$
\frac{\partial}{\partial t}\left(\begin{array}{c}
u_{n m} \\
\phi_{n m}^{\Delta}
\end{array}\right)=-\frac{\mathbf{c} \cdot \mathbf{a}}{\tau}\left(\begin{array}{c}
u_{n m} \\
\phi_{n m}^{\Delta}
\end{array}\right),
$$

for each $(n, m)$-mode. Here

$$
\tau=\frac{\eta^{+} r_{0}^{3}}{\kappa}
$$

is the bending relaxation time and the dimensionless matrices $\mathbf{a}$ and $\mathbf{c}$ are given by

$$
\begin{gathered}
\mathbf{a}=\left(\begin{array}{cc}
(n-1)(n+2)\left[n(n+1)+\sigma^{\prime}-2 \lambda^{\prime} \phi_{0}^{\Delta}+k^{\prime}\left(\phi_{0}^{\Delta}\right)^{2}\right] & 4 k^{\prime} \phi_{0}^{\Delta}-4 \lambda^{\prime}-\lambda^{\prime}(n-1)(n+2) \\
4 k^{\prime} \phi_{0}^{\Delta}-4 \lambda^{\prime}-\lambda^{\prime}(n-1)(n+2) & 2 k^{\prime}
\end{array}\right) \\
\mathbf{c}=\frac{n(n+1)}{d}\left(\begin{array}{cc}
4 w^{\prime}+(2 n+1)(1+E) & -(n+2)+(n-1) E \\
-(n+2)+(n-1) E & (n+2)\left(2 n^{2}-n+2\right)+(n-1)\left(2 n^{2}+5 n+5\right) E
\end{array}\right)
\end{gathered}
$$

where

$$
\begin{aligned}
d & =4 w^{\prime}\left[(n-1)\left(2 n^{2}+5 n+5\right) E+(n+2)\left(2 n^{2}-n+2\right)\right] \\
& +2(n-1)(n+1)\left(2 n^{2}+4 n+3\right) E^{2} \\
& +\left[8 n^{2}(n+1)^{2}-5\right] E+2 n(n+2)\left(2 n^{2}+1\right) .
\end{aligned}
$$

In the above matrices, the dimensionless parameters are defined as

$$
\begin{aligned}
\sigma^{\prime} & =\frac{\sigma r_{0}^{2}}{\kappa}, & \lambda^{\prime}=\frac{\lambda r_{0}}{\kappa}, & k^{\prime}=\frac{k r_{0}^{2}}{\kappa}, \\
w^{\prime} & =\frac{w r_{0}}{\eta^{+}}, & E & =\frac{\eta^{-}}{\eta^{+}} .
\end{aligned}
$$

The symmetric matrix a represents the inverse susceptibility and is composed of the static quantities. On the other hand, the matrix $\mathbf{c}$ is constructed by the dynamic quantities and corresponds to the kinetic coefficient matrix. Notice that both $\mathbf{a}$ and $\mathbf{c}$ are symmetric in accordance with the Onsager's reciprocal relation [26] and depend only on $n$.

In our previous work 25], we showed that the two relaxation modes are coupled to each other as a consequence of the bilayer nature and the spherical structure of the vesicle. We investigated the effect of viscosity contrast $E=\eta^{-} / \eta^{+}$on the relaxation rates, and found that it linearly shifts the crossover $n$-mode between the bending and the slipping relaxations. As $E$ is increased, the relaxation rate of the bending mode decreases, while that of the slipping mode remains almost unaffected. For parameter values close to the unstable region, some of the relaxation modes are dramatically reduced [25].

\section{THERMAL FLUCTUATIONS}

In this section, we discuss thermal fluctuations of a compressible bilayer vesicle. In the presence of random forces, the coupled memoryless Langevin equation for the two variables $u$ and $\phi^{\Delta}$ can be written as 26.

$$
\boldsymbol{\zeta} \cdot \frac{\partial}{\partial t} \mathbf{x}(t)=-\boldsymbol{\gamma} \cdot \mathbf{x}(t)+\boldsymbol{\xi}(t)
$$

where we have used the vector notation

$$
\mathbf{x}=r_{0}\left(\begin{array}{c}
u_{n m} \\
\phi_{n m}^{\Delta}
\end{array}\right)
$$

and the matrices

$$
\boldsymbol{\zeta}=\eta^{+} r_{0} \mathbf{c}^{-1}, \quad \gamma=\frac{\kappa}{r_{0}^{2}} \mathbf{a},
$$

where $\mathbf{c}^{-1}$ represents the inverse matrix. The vector $\mathbf{x}$ has the dimension of length in order to assign the proper dimension for each term in the above Langevin equation.

The vector $\boldsymbol{\xi}$ represents random forces acting directly 
TABLE I. Typical values for the physical quantities.

\begin{tabular}{ll}
\hline \hline bending modulus: $\kappa$ & $10^{-19} \mathrm{~J}$ \\
compressibility: $k$ & $10^{-1} \mathrm{~J} / \mathrm{m}^{2}$ \\
surface tension: $\sigma$ & $10^{-11} \mathrm{~J} / \mathrm{m}^{2}$ \\
outside solvent viscosity: $\eta^{+}$ & $10^{-3} \mathrm{~J} \cdot \mathrm{s} / \mathrm{m}^{3}$ \\
viscosity contrast: $E=\eta^{-} / \eta^{+}$ & 1,100 \\
inter-monolayer friction: $w$ & $10^{9} \mathrm{~J} \cdot \mathrm{s} / \mathrm{m}^{4}$ \\
vesicle radius: $r_{0}$ & $10^{-5} \mathrm{~m}$ \\
membrane relaxation time: $\tau=\eta^{+} r_{0}^{3} / \kappa$ & $10 \mathrm{~s}$ \\
activity strength: $S_{u} /\left(\eta^{+} r_{0}\right)$ & $80 k_{\mathrm{B}} T$ \\
activity relaxation time: $\tau_{u}$ & $10^{-1} \mathrm{~s}$ \\
\hline \hline
\end{tabular}

on $u$ and $\phi^{\Delta}$ :

$$
\boldsymbol{\xi}(t)=\left(\begin{array}{l}
\xi_{n m}^{u}(t) \\
\xi_{n m}^{\phi}(t)
\end{array}\right)
$$

In thermal equilibrium, these forces have zero mean and obey the following fluctuation dissipation theorem (FDT) [26]:

$$
\begin{aligned}
& \langle\boldsymbol{\xi}(t)\rangle=0, \\
& \left\langle\boldsymbol{\xi}\left(t_{1}\right) \boldsymbol{\xi}^{\mathrm{T}}\left(t_{2}\right)\right\rangle=2 k_{\mathrm{B}} T \boldsymbol{\zeta} \delta\left(t_{1}-t_{2}\right),
\end{aligned}
$$

where "T" indicates the transpose operator, $k_{\mathrm{B}}$ is the Boltzmann constant, and $T$ is the temperature. Notice that the friction matrix $\zeta$ (or $\mathbf{c}^{-1}$ ) contains off-diagonal elements, implying that thermal fluctuations of the two fields $u$ and $\phi^{\Delta}$ are correlated with each other. This is a unique feature of spherically closed vesicles because such a coupling effect does not exist for planar bilayer membranes [25].

The Fourier components of the $2 \times 2$ correlation function matrix can be obtained by

$$
\left(\mathbf{x x}^{\mathrm{T}}\right)_{\omega}=\int_{-\infty}^{\infty} d t\left\langle\mathbf{x}(t) \mathbf{x}^{\mathrm{T}}(0)\right\rangle e^{i \omega t}
$$

where $\omega$ is the frequency. With the use of Eqs. (18) and (19), the thermal contribution to the correlation function matrix is given by 31 .

$$
\left(\mathbf{x x}^{\mathrm{T}}\right)_{\omega}^{\mathrm{th}}=2 k_{\mathrm{B}} T(\boldsymbol{\gamma}-i \omega \boldsymbol{\zeta})^{-1} \cdot \boldsymbol{\zeta} \cdot(\boldsymbol{\gamma}+i \omega \boldsymbol{\zeta})^{-1}
$$

whose components depend on $n$. Then the power spectral density (PSD) matrix is obtained by summing over all the $n$-modes as

$$
\begin{aligned}
\mathbf{P}_{\omega}^{\mathrm{th}} & =\left(\begin{array}{cc}
\left(u^{2}\right)_{\omega}^{\mathrm{th}} & (u \phi)_{\omega}^{\mathrm{th}} \\
(\phi u)_{\omega}^{\mathrm{th}} & \left(\phi^{2}\right)_{\omega}^{\mathrm{th}}
\end{array}\right) \\
& =\sum_{n=2}^{n_{\max }}(2 n+1)\left(\mathbf{x x}^{\mathrm{T}}\right)_{\omega}^{\mathrm{th}} .
\end{aligned}
$$

Notice that the components of the correlation function matrix do not depend on $m$ but are $(2 n+1)$-fold degenerated. In the following numerical estimate, we set

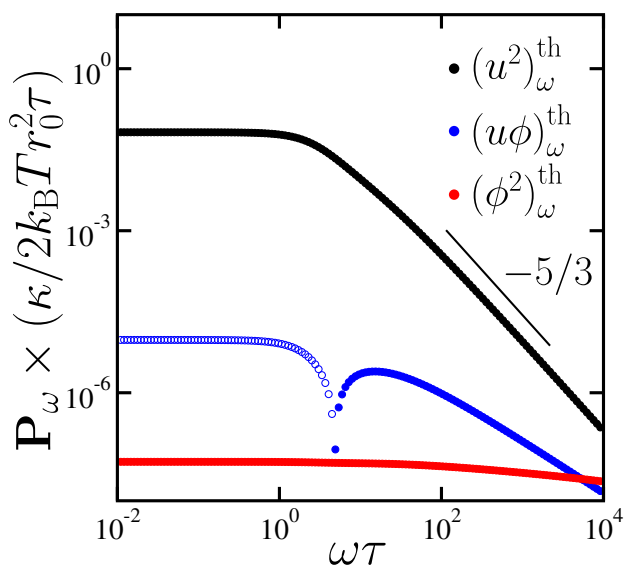

FIG. 2. Dimensionless components of thermal power spectral density (PSD) matrix as a function of dimensionless frequency $\omega \tau$. The characteristic bending relaxation time is $\tau=\eta^{+} r_{0}^{3} / \kappa$ which is chosen here as $\tau=10 \mathrm{~s}$ (see Table I). The dimensionless parameter values are $\sigma^{\prime}=10^{-2}, \lambda^{\prime}=10^{4}, k^{\prime}=10^{8}$, $w^{\prime}=10^{7}, \phi_{0}^{\Delta}=3 \times 10^{-4}, E=1$ (these values can be obtained from Table I). Three independent components are plotted; $\left(u^{2}\right)_{\omega}^{\text {th }}$ (black), $(u \phi)_{\omega}^{\text {th }}$ (blue), and $\left(\phi^{2}\right)_{\omega}^{\text {th }}$ (red). The component $(u \phi)_{\omega}^{\text {th }}$ becomes negative for small frequencies as represented by open blue circles. The slope of $-5 / 3$ is drawn for comparison.

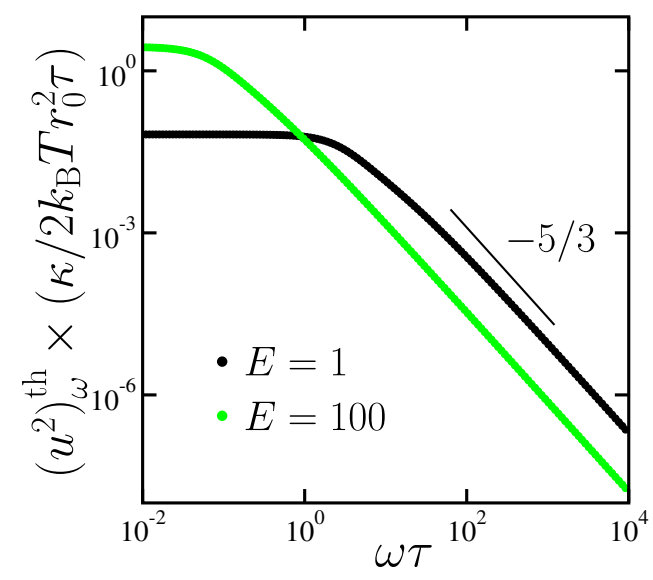

FIG. 3. Dimensionless thermal power spectral density $\left(u^{2}\right)_{\omega}^{\text {th }}$ as a function of dimensionless frequency $\omega \tau$ for $E=1$ (black) and $E=100$ (green). The other parameters are the same with those in Fig. 2 The slope of $-5 / 3$ is drawn for comparison.

$n_{\max }=1000$ which covers sufficient length scales for the present analysis.

In Fig. 2, we plot the frequency dependence of each component of the numerically calculated PSD matrix in Eq. (22) for a vesicle of size $r_{0}=10 \mu \mathrm{m}$. The other parameter values and the relevant quantities are summarized in Table I. The three curves correspond to the three components of the symmetric PSD matrix $\left(u^{2}\right)_{\omega}^{\text {th }}$ (black), $(u \phi)_{\omega}^{\text {th }}$ (blue), and $\left(\phi^{2}\right)_{\omega}^{\text {th }}$ (red). Within the present scaled unit, $\left(u^{2}\right)_{\omega}^{\text {th }}$ is much larger than $\left(\phi^{2}\right)_{\omega}^{\text {th }}$ and $(u \phi)_{\omega}^{\text {th }}$. Hence the density field fluctuations are relatively small. The 
PSD component $(u \phi)_{\omega}^{\text {th }}$ lies in between $\left(u^{2}\right)_{\omega}^{\text {th }}$ and $\left(\phi^{2}\right)_{\omega}^{\text {th }}$, and takes negative values in the small frequency region (shown by open blue circles).

In general, all the PSD components are almost constant in the low-frequency regime and decay in the highfrequency regime. The important crossover frequency $\omega^{*}$ is set by the membrane relaxation time $\tau=\eta^{+} r_{0}^{3} / \kappa$ and is given by $\omega^{*} \approx 1 / \tau$. For the parameter values in Table $\mathrm{I}$, the characteristic relaxation time is chosen as $\tau=10 \mathrm{~s}$ and $\omega^{*}=0.1 \mathrm{~s}^{-1}$. In the low frequency regime of $\omega \tau \ll 1$ (or $\omega \ll \omega^{*}$ ), all the PSD components become independent of the frequency, which is a characteristic feature of a vesicle with a finite size. In the high frequency limit of $\omega \tau \gg 1$ (or $\left.\omega \gg \omega^{*}\right)$, the PSD $\left(u^{2}\right)_{\omega}^{\mathrm{th}}$ shows a power-law decay with an exponent -1.60 which is close to the exponent $-5 / 3$ obtained by Zilman and Granek for a flat membrane without any bilayer structure [32, 33]. We also note that the frequency dependence of $\left(\phi^{2}\right)_{\omega}^{\text {th }}$ is very weak in the entire region. Hence, we shall mainly focus on the behavior of displacement fluctuations $\left(u^{2}\right)_{\omega}$ in the following discussion.

To see the effect of viscosity contrast between the inside and the outside of a vesicle, we plot in Fig. 3 the PSD component $\left(u^{2}\right)_{\omega}^{\text {th }}$ for $E=\eta^{-} / \eta^{+}=1$ (the same as in Fig. 21) and $E=100$ (large inside viscosity [34]). When the inside viscosity becomes larger, the crossover frequency $\omega^{*}$ becomes smaller and the fluctuation amplitude is enhanced in the low-frequency region. This is because the effective viscosity for $E \neq 1$ is essentially given by the combination of the two viscosities such that $\eta_{\text {eff }} \approx \eta^{+}+\eta^{-}$. In the high frequency regime, on the other hand, the PSD for $E=100$ is smaller than that of $E=1$ because the displacement fluctuations are suppressed when the inner viscosity is larger. However, the power-law behavior in the large frequency limit $\left(\omega \gg \omega^{*}\right)$ of the PSD with an exponent $-1.6 \approx-5 / 3$ is the same for the two cases of $E=1$ and 100 .

\section{ACTIVE FLUCTUATIONS}

In this section, we discuss the influence of nonequilibrium random forces on the power spectrum of fluctuations. In real cells, these active forces are generated as a consequence of metabolic processes such as the pumping action of ion channels or the growth of cytoskeletal networks [9]. The modified coupled Langevin equation in the presence of active random forces is given by

$$
\boldsymbol{\zeta} \cdot \frac{\partial}{\partial t} \mathbf{x}(t)=-\boldsymbol{\gamma} \cdot \mathbf{x}(t)+\boldsymbol{\xi}(t)+\boldsymbol{\mu}(t)
$$

where we have added the following vector of active random forces to Eq. (14);

$$
\boldsymbol{\mu}(t)=\left(\begin{array}{c}
\mu_{n m}^{u}(t) \\
\mu_{n m}^{\phi}(t)
\end{array}\right) .
$$
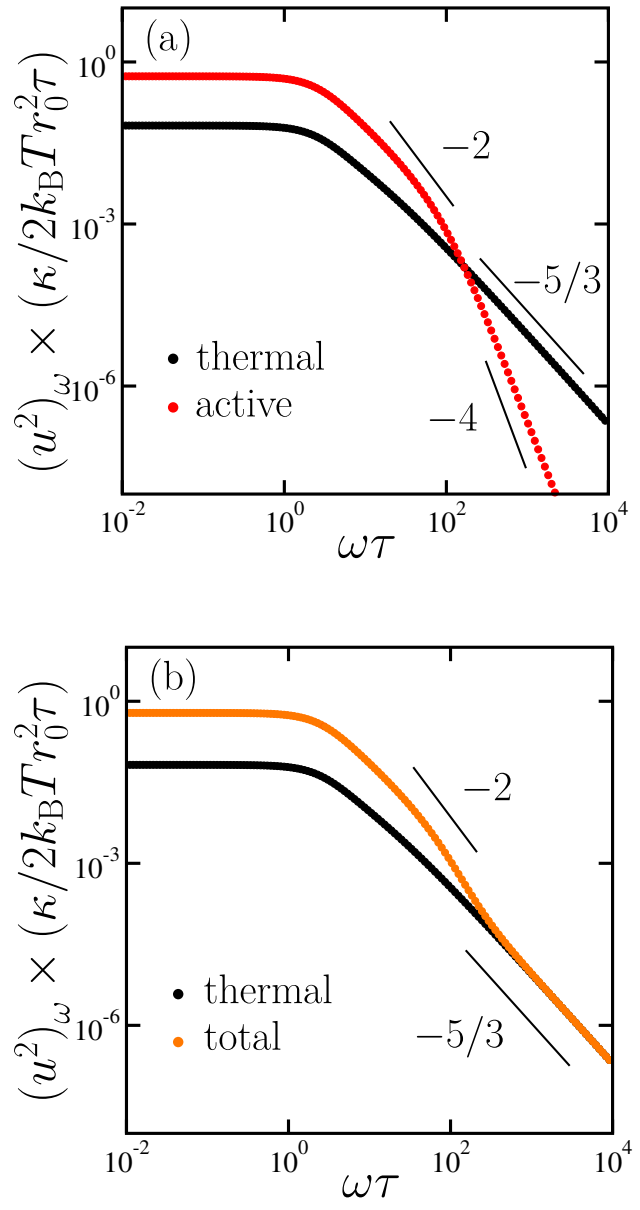

FIG. 4. (a) Dimensionless thermal power spectral density $\left(u^{2}\right)_{\omega}^{\text {th }}$ (black) and active power spectral density $\left(u^{2}\right)_{\omega}^{\text {ac }}$ (red) as a function of dimensionless frequency $\omega \tau$. The parameters for the active contribution are $S_{u} /\left(\eta^{+} r_{0}\right)=80 k_{\mathrm{B}} T$, $\tau_{u} / \tau=10^{-2}, S_{\phi}=0$ (see Table I). The other parameters are the same with those in Fig. 2 (b) Dimensionless thermal power spectral density $\left(u^{2}\right)_{\omega}^{\text {th }}$ (black) and total power spectral density $\left(u^{2}\right)_{\omega}=\left(u^{2}\right)_{\omega}^{\text {th }}+\left(u^{2}\right)_{\omega}^{\text {ac }}$ (orange) as a function of dimensionless frequency $\omega \tau$. The slopes of $-5 / 3,-2$, and -4 are drawn for comparison.

We assume that these random forces have zero mean and their cross correlations vanish, i.e.,

$$
\begin{aligned}
& \langle\boldsymbol{\mu}(t)\rangle=0, \\
& \left\langle\mu_{n m}^{u}\left(t_{1}\right) \mu_{n^{\prime} m^{\prime}}^{\phi}\left(t_{2}\right)\right\rangle=0 .
\end{aligned}
$$

In the above, active fluctuations operate directly on the displacement and the density fields. They can be used to model any of the several metabolic processes that modify the membrane dynamics. For instance, nonequilibrium fluctuations acting on the displacement field mimic processes that affect the membrane dynamics in the radial direction such as pumping of ion channels or cytoskeletal growth. On the other hand, fluctuations acting on the density field can represent the action of flippase proteins which consume ATP and facilitate exchange of lipid molecules between the two monolayers. 


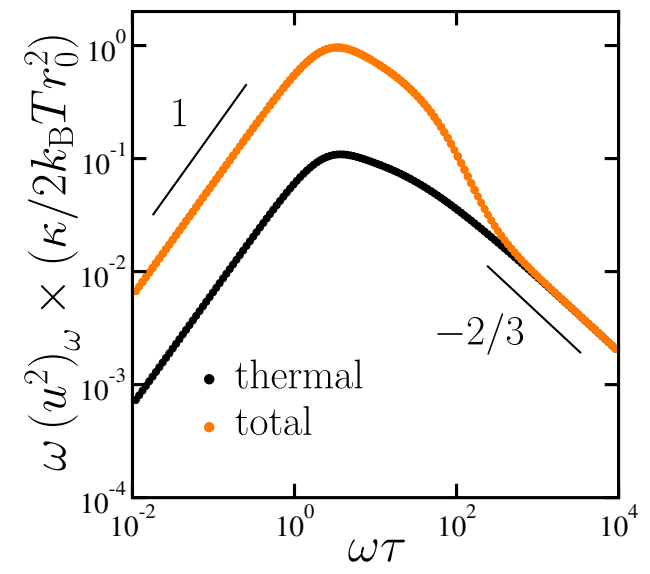

FIG. 5. Dimensionless thermal response function $\omega\left(u^{2}\right)_{\omega}^{\text {th }} /\left(2 k_{\mathrm{B}} T\right)$ (black) and total apparent response function $\omega\left(u^{2}\right)_{\omega} /\left(2 k_{\mathrm{B}} T\right)=\left[\left(u^{2}\right)_{\omega}^{\mathrm{th}}+\left(u^{2}\right)_{\omega}^{\mathrm{ac}}\right] /\left(2 k_{\mathrm{B}} T\right)$ (orange) as a function of dimensionless frequency $\omega \tau$. The other parameters are the same with those in Fig. 4 The slopes of $-2 / 3$ and 1 are drawn for comparison.

In general, the membrane dynamics can be affected by several active processes with different characteristic timescales. For simplicity, however, we consider the case when there is only one dominant active process characterized by a single timescale for all the modes. Then the correlations of active random forces are assumed to have the forms

$$
\begin{aligned}
& \left\langle\mu_{n m}^{u}\left(t_{1}\right) \mu_{n^{\prime} m^{\prime}}^{u}\left(t_{2}\right)\right\rangle=\frac{S_{u}}{2 \tau_{u}} e^{-\left|t_{1}-t_{2}\right| / \tau_{u}} \delta_{n n^{\prime}} \delta_{m m^{\prime}}, \\
& \left\langle\mu_{n m}^{\phi}\left(t_{1}\right) \mu_{n^{\prime} m^{\prime}}^{\phi}\left(t_{2}\right)\right\rangle=\frac{S_{\phi}}{2 \tau_{\phi}} e^{-\left|t_{1}-t_{2}\right| / \tau_{\phi}} \delta_{n n^{\prime}} \delta_{m m^{\prime}},
\end{aligned}
$$

where $\tau_{u}$ and $\tau_{\phi}$ are the characteristic time scales, while $S_{u}$ and $S_{\phi}$ denote the strengths of the activity. Here we also assume that these quantities depend neither on $n$ nor on $m$.

The Fourier transform of the above active force correlations can be expressed in the matrix form as

$$
\left(\boldsymbol{\mu} \boldsymbol{\mu}^{\mathrm{T}}\right)_{\omega}=\left(\begin{array}{cc}
\frac{S_{u}}{1+\left(\omega \tau_{u}\right)^{2}} & 0 \\
0 & \frac{S_{\phi}}{1+\left(\omega \tau_{\phi}\right)^{2}}
\end{array}\right),
$$

where the diagonal elements are Lorentzian functions. Using this expression, we can write the active contribution to the correlation function matrix as

$$
\left(\mathbf{x x}^{\mathrm{T}}\right)_{\omega}^{\mathrm{ac}}=(\boldsymbol{\gamma}-i \omega \boldsymbol{\zeta})^{-1} \cdot\left(\boldsymbol{\mu} \boldsymbol{\mu}^{\mathrm{T}}\right)_{\omega} \cdot(\boldsymbol{\gamma}+i \omega \boldsymbol{\zeta})^{-1}
$$

whose components depend only on $n$. Then the active PSD matrix can be calculated by

$$
\begin{aligned}
\mathbf{P}_{\omega}^{\mathrm{ac}} & =\left(\begin{array}{cc}
\left(u^{2}\right)_{\omega}^{\mathrm{ac}} & 0 \\
0 & \left(\phi^{2}\right)_{\omega}^{\mathrm{ac}}
\end{array}\right) \\
& =\sum_{n=2}^{n_{\max }}(2 n+1)\left(\mathbf{x x}^{\mathrm{T}}\right)_{\omega}^{\mathrm{ac}},
\end{aligned}
$$

similar to the thermal contribution in Eq. (22). Finally, the total PSD is given by the sum of thermal and active contributions;

$$
\mathbf{P}_{\omega}=\mathbf{P}_{\omega}^{\mathrm{th}}+\mathbf{P}_{\omega}^{\mathrm{ac}} .
$$

Here we have implicitly assumed that thermal and active random forces are not correlated with each other.

In Fig. $4(\mathrm{a})$, we plot both the thermal PSD component $\left(u^{2}\right)_{\omega}^{\text {th }}$ (black, the same as in Fig. 2) and the active PSD component $\left(u^{2}\right)_{\omega}^{\mathrm{ac}}$ (red) as a function of $\omega \tau$ when $S_{u} /\left(\eta^{+} r_{0}\right)=80 k_{\mathrm{B}} T, \tau_{u} / \tau=10^{-2}$, and $S_{\phi}=0$ neglecting active fluctuations of the density field $\phi^{\Delta}$. Since the membrane relaxation time is chosen as $\tau=10 \mathrm{~s}$ for a vesicle of size $r_{0}=10 \mu \mathrm{m}$, the above parameter means that the activity time scale is roughly $\tau_{u}=10^{-1} \mathrm{~s}[9]$. The active contribution decays as $\sim \omega^{-2}$ in the intermediate frequency regime, $\tau^{-1} \ll \omega \ll \tau_{u}^{-1}$, and further decays as $\sim \omega^{-4}$ in the high frequency region, $\omega \tau_{u} \gg 1$. In Fig. 4(b), we plot the total PSD component $\left(u^{2}\right)_{\omega}$ (orange) given by Eq. (32) as well as the thermal PSD (black, the same as in (a)) in order to compare between the equilibrium and nonequilibrium situations. We note that active contribution becomes important in the low frequency region $\omega \tau_{u} \ll 1$ and enhances the total fluctuations in the nonequilibrium active case.

To present our result in a different way, we plot in Fig. [5 an apparent total response function defined by $\omega \mathbf{P}_{\omega} /\left(2 k_{\mathrm{B}} T\right)$ (orange) as a function of $\omega \tau$. We use the

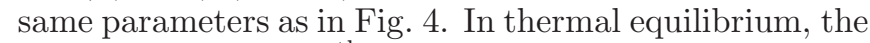
response function $\omega \mathbf{P}_{\omega}^{\text {th }} /\left(2 k_{\mathrm{B}} T\right)$ (black) should satisfy the relation [31]

$$
\frac{\omega \mathbf{P}_{\omega}^{\mathrm{th}}}{2 k_{\mathrm{B}} T}=\chi^{\prime \prime}(\omega)
$$

where $\chi^{\prime \prime}(\omega)$ is the imaginary part of the complex response function matrix given by

$$
\chi(\omega)=\sum_{n=2}^{n_{\max }}(2 n+1)(\gamma-i \omega \boldsymbol{\zeta})^{-1} .
$$

Here the response function matrix can be obtained from Eq. (23) when both thermal and active fluctuations are absent, i.e., $\boldsymbol{\xi}=\boldsymbol{\mu}=0$. In nonequilibrium case, the apparent response function $\omega \mathbf{P}_{\omega} /\left(2 k_{\mathrm{B}} T\right)$ (orange) is enhanced. Moreover, it increases linearly with $\omega$ in the low frequency regime, while it exhibits a power-law decay with an exponent $-2 / 3$ in the high frequency regime. As we shall discuss in the next section, these results are consistent with the experimental findings by Turlier et al. 9] who used a more detailed model to take into account the activity.

Generally speaking, Eq. (33) is an alternative expression of the FDT [31]. It states that the thermal PSD matrix $\mathbf{P}_{\omega}^{\text {th }}$ and the imaginary part of the response function $\chi^{\prime \prime}(\omega)$ are intimately related in equilibrium. In the presence of active fluctuations, the total PSD of fluctuations includes both thermal and active contributions $\mathbf{P}_{\omega}^{\text {th }}+\mathbf{P}_{\omega}^{\mathrm{ac}}$ 
as in Eq. (32), whereas an experimentally measured response function should not be altered even in nonequilibrium situations. Therefore, the FDT is inevitably violated in the presence of active fluctuations, and the extent of violation is generally characterized by $\mathbf{P}_{\omega}^{\mathrm{ac}}$.

\section{SUMMARY AND DISCUSSIONS}

In this paper, we have discussed the statistical properties of thermal and active fluctuations of a compressible bilayer vesicle. We have analyzed the coupled Langevin equation for the membrane deformation and the lipid density variables. In particular, we have calculated the PSD matrix of these fluctuations and discussed the frequency dependence of each component. Thermal contribution to PSD is estimated by means of the FDT, whereas active contribution is obtained by assuming that the time correlation functions of active random forces decay exponentially with a characteristic time scale. We have assumed that the total PSD is given by the sum of thermal and active contributions. Our results show that the nonequilibrium active contribution affects fluctuations below a characteristic frequency set by the activity timescale. To compare with the recent microrheology experiment on RBCs [9], we have further obtained an apparent response function which is enhanced in the low-frequency regime.

Recently, Turlier et al. reported a FDT violation and argued that the cell membrane fluctuations in healthy RBCs are actively driven [9]. The experimentally measured fluctuations and response function were in agreement with their theoretical calculations obtained from the spectrin network model of active membranes. They showed that the timescale of switching between active and inactive states of the phosphorylation sites on the spectrin network is roughly $0.15 \mathrm{~s}[9]$. In order to make a comparison with their experiment, we have set the activity timescale $\tau_{u}=0.1 \mathrm{~s}$ to calculate the apparent response function in Fig. 5. We have shown that the enhanced response function increases linearly at low frequencies $\omega \tau \ll 1$ and decays as $\sim \omega^{-2 / 3}$ at very high frequencies $\omega \tau_{u} \gg 1$. These results are in accordance with the experimental result by Turlier et al. [9].

Most of the existing works on membrane fluctuations describe only the dynamics of the displacement field $u$. In this work, we have taken into account the compressibility of the membrane and have also discussed the dynamics of the lipid density field $\phi^{\Delta}$. In order to write down the coupled Langevin equation in Eq. (14), we have used the fact that the dynamics of these two variables is expressed by the product of the two symmetric matrices, i.e., the inverse susceptibility matrix a in Eq. (9) and the kinetic coefficient matrix $\mathbf{c}$ in Eq. (10). The obtained PSD matrix characterizes the frequency dependencies of fluctuation amplitudes for the membrane displacement and the lipid density. Although some works have reported the effects of bilayer nature on the static fluctuation spec- trum of the membrane displacement [35, 36], we are not aware of any experimental work where the lipid density fluctuation is investigated explicitly.

The thermal PSD component $\left(u^{2}\right)_{\omega}^{\text {th }}$ is related to the Fourier transform of the mean squared displacement (MSD) of a tagged membrane segment. This is because the MSD and the displacement correlation function are related by $\left\langle[u(t)-u(0)]^{2}\right\rangle=2\left[\left\langle u^{2}(0)\right\rangle-\langle u(t) u(0)\rangle\right]$ and the time dependencies of the MSD and the correlation function are the same except for the numerical prefactor (as long as the equal time correlation function $\left\langle u^{2}(0)\right\rangle$ is finite) [26]. For a flat membrane, Zilman and Granek predicted that a membrane segment undergoes anomalous out-of-plane diffusion for which the MSD increases as $\sim t^{2 / 3}[32,33]$. In the frequency domain, this behavior is consistent with our result of $\left(u^{2}\right)_{\omega}^{\text {th }} \sim \omega^{-5 / 3}$ as shown in Fig. 2. Therefore, we confirm that the scaling relation for a flat membrane also holds for a compressible bilayer vesicle. Moreover, we find that this scaling behavior is not affected by the viscosity contrast $E$ as shown in Fig. 3. The thermal PSD component $\left(u^{2}\right)_{\omega}^{\mathrm{th}}$ is independent of $\omega$ at low frequencies $\left(\omega \tau \ll 1\right.$ or $\left.\omega \ll \omega^{*}\right)$ because the slowest bending relaxation time $\tau$ is set by the finite size of a vesicle.

In our theory, both the inertia of the membrane segment and the inertia of the surrounding fluid are neglected when we calculate the hydrodynamic response of a bilayer vesicle. Within this approximation, we have employed the standard memoryless Langevin equation in the overdamped form. On the other hand, it is known that the Brownian motion of a spherical particle in an incompressible fluid should be described by the generalized Langevin equation [37] with the Boussinesq force 38]. For a spherical particle in a $3 \mathrm{D}$ fluid, the correction to the Stokes friction due to the fluid inertia (called the Basset force) leads to a long-time tail behavior of the velocity autocorrelation function (typically $\sim t^{-3 / 2}$ ) [39, 40]

It is beyond the scope of the present paper to calculate the hydrodynamic memory effect of fluid inertia on the membrane friction matrix $\boldsymbol{\zeta}$ [see Eq. (16)]. However, we can roughly estimate the crossover frequency $\omega^{* *}$ above which the fluid inertia plays a role. The minimum of the crossover frequency is roughly given by $\omega^{* *}=\eta^{+} /\left(\rho r_{0}^{2}\right)$, where $\eta^{+}$is the fluid viscosity, $\rho$ is the fluid density, and $r_{0}$ corresponds to the vesicle radius which is the largest size in the problem. With the value $\rho \approx 10^{3} \mathrm{~kg} / \mathrm{m}^{3}$ for water and the other values quoted in Table I, we obtain $\omega^{* *} \approx 10^{4} \mathrm{~s}^{-1}$ and $\omega^{* *} \tau \approx 10^{5}$, where $\tau \approx 10 \mathrm{~s}$ is the bending relaxation time of the vesicle [see Eq. [8] ]. Generally, inertial effects of the fluid become strong at frequencies higher than $\omega^{* *}$. In other words, effects of fluid inertia and stress propagation are negligible for frequencies $\omega \ll \omega^{* *}$. Furthermore, an analogous crossover frequency for a membrane segment (rather than a whole vesicle) can be even larger because its size is much smaller than the vesicle size $r_{0}$.

The frequency range considered in Figs. 215 is $10^{-2} \leq$ $\omega \tau \leq 10^{4}$ for which we are safely allowed to neglect in- 
ertial effects of the surrounding bulk fluid. Although our calculation relies on the Langevin equation without any hydrodynamic memory effect, the "large frequency" regime in the graphs $\left(1 \ll \omega \tau \leq 10^{4}\right)$ corresponds to the "small frequency" regime when compared with the above estimated crossover frequency $\omega^{* *} \tau \approx 10^{5}$ related to the fluid inertia. This argument justifies the usage of the standard Langevin equation and allows us to discuss the long time behavior of membrane fluctuations. We also comment that the frequency range $10^{-3}-10^{3} \mathrm{~s}^{-1}$ plotted in the graphs are easily accessible by the current experimental techniques.

Here we shall briefly mention that both the inertia of the fluid membrane and the surrounding fluid were considered by Camley and Brown [4] and by Komura et al. [42, 43] for an infinitely large and flat membrane without any out-of-plane deformations. Especially, Camley and Brown showed in the former paper that fluid inertia creates a long-time tail in the membrane velocity correlation function. In a viscous membrane, this tail crosses over from $\sim t^{-1}$ (as in a $2 \mathrm{D}$ fluid) at intermediate times to $\sim t^{-3 / 2}$ (as in a 3D fluid) at long times. An analogous study for membranes undergoing out-of-plane fluctuations can be interesting. However, it was also pointed out that a long-time tail behavior in a membrane becomes important only when the correlation function reaches $10^{-6}$ of its initial value, which would be effectively unobservable for vesicles [41].

On the other hand, the memory effect due to the viscoelasticity of the surrounding media is worth considering. When the outer fluid is viscoelastic, the constant viscosity $\eta$ in the Stokes equation should be replaced by a frequency dependent viscosity $\eta(\omega)$ in Eq. (16) according to the correspondence principle of linear viscoelasticity [44, 45]. Granek used a generalized Langevin equa- tion with a memory kernel to calculate the membrane MSD [46]. He also showed that, if the complex shear viscosity of the fluid is described by a power-law behavior $\eta(\omega) \sim(i \omega)^{\alpha-1}$, a tagged membrane segment would exhibit an anomalous subdiffusive behavior and its MSD should grow as $\sim t^{2 \alpha / 3}$. For $\alpha=1$, we recover the scaling behavior for a purely viscous medium as described before. Thus, we expect the PSD to scale as $\sim \omega^{-(2 \alpha+3) / 3}$ when the vesicle is surrounded by a viscoelastic medium which can mediate mechanical memory effects.

Finally, it is worth mentioning that we have chosen simple forms for active noise correlations that capture only limited features of active processes such as their timescale and strength. As an extension of the present work, we shall consider a coupling effect between active fluctuations and membrane local curvature. Such a coupling can be taken into account through a wavenumber dependent activity strength [17], and it will alter the frequency dependence of active fluctuations. In general, the frequency dependency of PSD can be used to characterize and distinguish between different active noise sources in experiments.

\section{ACKNOWLEDGMENTS}

T.V.S.K. acknowledges Tokyo Metropolitan University for the support provided through the co-tutorial program. S.K. and R.O. acknowledge support by Grant-inAid for Scientific Research on Innovative Areas "Fluctuation and Structure" (Grant No. 25103010) from the Ministry of Education, Culture, Sports, Science, and Technology (MEXT) of Japan and by Grant-in-Aid for Scientific Research (C) (Grant No. 15K05250) from the Japan Society for the Promotion of Science (JSPS).
[1] S. A. Safran, Statistical Thermodynamics of Surfaces, Interfaces, and Membranes (Addison-Wesley, Reading, MA, 1994).

[2] R. Rodríguez-García, L. R. Arriaga, M. Mell, L. H. Moleiro, I. López-Montero, and F. Monroy, Phys. Rev. Lett. 102, 128101 (2009).

[3] D. Boss, A. Hoffmann, B. Rappaz, C. Depeursinge, P. J. Magistretti, D. V. de Ville, and P. Marquet, PLoS ONE 7, e40667 (2012).

[4] J. F. Faucon, M. D. Mitov, P. Méléard, I. Bivas, and P. Bothorel, J. Phys. (Paris) 50, 2389 (1989).

[5] S. T. Milner and S. A. Safran, Phys. Rev. A 36, 4371 (1987).

[6] F. Brochard and J. F. Lennon, J. Phys. France 36, 1035 (1975).

[7] T. Betz, M. Lenz, J.-F. Joanny, and C. Sykes, Proc. Natl Acad. Sci. USA 106, 15320 (2009).

[8] Y. Park, C. A. Best, T. Auth, N. S. Gov, S. A. Safran, G. Popescu, S. Suresh, and M. S. Feld, Proc. Natl Acad. Sci. USA 107, 1289 (2010).
[9] H. Turlier, D. A. Fedosov, B. Audoly, T. Auth, N. S. Gov, C. Sykes, J.-F. Joanny, G. Gompper, and T. Betz, Nature Physics 12, 513 (2016).

[10] A. Biswas, A. Alex, and B. Sinha, Biophys. J. 113, 1768 (2017).

[11] V. G. Almendro-Vedia, P. Natale, M. Mell, S. Bonneau, F. Monroy, F. Joubert, and I. López-Montero, Proc. Natl Acad. Sci. 114, 11291 (2017).

[12] S. F. Fenz, T. Bihr, D. Schmidt, R. Merkel, U. Seifert, K. Sengupta, and A. Smith, Nature Physics 13, 906 (2017).

[13] J. Prost and R. Bruinsma, Europhys. Lett. 33, 321 (1996).

[14] S. Ramaswamy, J. Toner, and J. Prost, Phys. Rev. Lett. 84, 3494 (2000).

[15] D. Lacoste and A. W. C. Lau, Europhys. Lett. 70 (3), 418 (2005).

[16] S. Komura, K. Yasuda, and R. Okamoto, J. Phys.: Condens. Matter 27, 432001 (2015).

[17] N. Gov, Phys. Rev. Lett. 93, 268104 (2004).

[18] N. S. Gov and S. A. Safran, Biophys. J. 88, 1859 (2005).

[19] N. S. Gov, Phys. Rev. E 75, 011921 (2007). 
[20] K. Yasuda, S. Komura, and R. Okamoto, Phys. Rev. E 93, 052407 (2016).

[21] U. Seifert and S. A. Langer, Europhys. Lett. 23, 71 (1993).

[22] L. Miao, M. A. Lomholt, and J. Kleis, Eur. Phys. J. E 9, 143 (2002).

[23] J.-B. Fournier, Int. J. Nonlinear Mech. 75, 67 (2015).

[24] R. Okamoto, Y. Kanemori, S. Komura, and J.-B. Fournier, Eur. Phys. J. E 39, 52 (2016).

[25] T. V. Sachin Krishnan, R. Okamoto, and S. Komura, Phys. Rev. E 94, 062414 (2016).

[26] M. Doi, Soft Matter Physics (Oxford University, Oxford, 2013).

[27] R. Okamoto, S. Komura, and J.-B. Fournier, Phys. Rev. E 96, 012416 (2017).

[28] S. Komura and K. Seki, Physica A 192, 27 (1993).

[29] K. Seki and S. Komura, Physica A 219, 253 (1995).

[30] S. Komura, in Vesicles, edited by M. Rosoff (Marcel Dekker, Inc., New York, 1996), p. 198.

[31] L. D. Landau and E. M. Lifshitz, Statistical Physics (Pergamon Press, Oxford, 1980).

[32] A. G. Zilman and R. Granek, Phys. Rev. Lett. 77, 4788 (1996).

[33] A. G. Zilman and R. Granek, Chem. Phys. 284, 195 (2002).

[34] K. Fujiwara and M. Yanagisawa, ACS Synth. Biol. 3, 870 (2014).
[35] M. C. Watson and F. L. H. Brown, Biophys. J 98, L09 (2010).

[36] M. Mell, L. H. Moleiro, Y. Hertle, I. López-Montero, F. J. Cao, P. Fouquet, T. Hellweg, and F. Monroy, Chem. Phys. Lipids 185, 61 (2015).

[37] R. Kubo, M. Toda, and N. Hashitsume, Statistical Physics II (Springer-Verlag, New York, 1991).

[38] L. D. Landau and E. M. Lifshitz, Fluid Mechanics (Pergamon Press, Oxford, 1987).

[39] T. Franosch, M. Grimm, M. Belushkin, F. M. Mor, G. Foffi, L. Forró, and S. Jeney, Nature 478, 85 (2011).

[40] S. Kheifets, A. Simha, K. Melin, T. Li, and M. G. Raizen, Science 343, 1493 (2014).

[41] B. A. Camley and F. L. H. Brown, Phys. Rev. E 84, 021904 (2011).

[42] K. Seki and S. Komura, Phys. Rev. E 47, 2377 (1993).

[43] S. Komura, S. Ramachandran, and K. Seki, EPL 97, 68007 (2012).

[44] M. Grimm, S. Jeney, and T. Franosch, Soft Matter 7, 2076 (2011).

[45] E. M. Furst and T. M. Squiers, Microrheology (Oxford University Press, Oxford, 2017).

[46] R. Granek, Soft Matter 7, 5281 (2011). 